\title{
Do Not Resuscitate Expressly Allowed Natural Death: A Common Ethical Dilemma Among Terminally Ill Cancer Patients
}

\author{
Nezar Ahmed Salim ${ }^{*}$, Roxanne Nematollahi ${ }^{2}$, Hasan Abu Nigim ${ }^{1}$ and Adam Tamimi ${ }^{3}$ \\ ${ }^{1}$ Dubai Health Authority (DHA), Dubai hospital, United Arab Emirates, Dubai \\ ${ }^{2}$ Medical Education Department, Dubai Health Authority, United Arab Emirates, Dubai \\ ${ }^{3}$ American hospital, Oncology $\backslash$ Hematology Clinic, United Arab Emirates, Dubai
}

\begin{abstract}
Background: Every human being desires a life without suffering and to live his or her life in best possible quality of life, but when death is looming by a terminal disease and one is lying in bed with machines to maintain organ functions; there comes the dilemma of the choice. However, patients may place healthcare providers at prospect of ethical dilemma by declining to care or to provide treatment, despite that patients have the right to do so.

Aim: This paper examines the ongoing and controversial debate regarding the DNR orders. It discusses the rights of terminally ill patients who opt to refuse treatment as well as the various legal and moral ramifications surrounding this particular topic.

Method: In this argumentative paper search was conducted using the electronic databases of CINAHL, Medline, PubMed, EPSCO, and Science Direct for articles published between 1994 to 2015. Twenty articles were found after extensive searching via electronic databases. There is no ethical approval to this article.

Results: Number of studies supported the DNR order to for terminally ill patients to allow them to pass away in peace. Also CPR in many cases may not end up with direct clinical benefits as the resuscitation could not be fruitful or lead to complications, which in result will extend suffering without treating the underlying illness.

Conclusion and recommendations: DNR should be taken into consideration especially with patients who have exhausted all other kind of treatment modalities in which there is multi-organ failure and hope for cure has been faded. The DNR as a concept may appear harsh and unacceptable for the patient and his loved ones. The use of this term or language plays an important role. The authors strongly believe that it would be more appropriate if the wording is changed from "Do Not Resuscitate" to "Allow Natural Death."
\end{abstract}

\section{Introduction}

The definition of death in Black low dictionary is the irreversible cessation of functions of circulation, pulsation, and respiration, it is also the absence of human activities when absent brain activities show a flat electroencephalogram for 48 hours [1].

Every human being desires a life without suffering and to live with the best possible quality of life, but when death is looming because of a terminal disease and one is lying in bed with machines to maintain organ functions; there comes the dilemma of the choice. Health care providers resort to Cardiopulmonary Resuscitation (CPR) as a last choice, so when (CPR) appears to be the only option to rescue the life of a terminally ill patient, many medical interventions can be perceived as pointless by the patient's family and health care providers. There is no denial that technology has advanced the medical field to an extent that prolongs life, although not necessarily the quality of it; with more regard to medical economics and less toward what 'end of life' preferences were made by patient's family or an advance directive.

However, many factors affect End of Life practices, this include physicians' personal attitudes, culture, family decisions, ethics, religious beliefs, financial status, hospital policy and country legislation might act as a life and cost saving approach [2].

The appropriate action and decision to take is an issue of ethical dilemmas. This happens among health care providers when they come to choose between two or more satisfactory courses of action, or evenly undesirable alternatives. Modern breakthroughs in treatment and technology have allowed healthcare professionals to prolong life expectancy. However, patients may place healthcare providers at the prospect of an ethical dilemma by declining care or treatment, despite the patients the right to do so.

Each person should consider having advanced directives present which could make complex End of Life situations less complicated for healthcare workers, patients and their families. Whether to start or not to start with CPR is the main topic of this paper in which is "Do Not Resuscitate (DNR)" order.

In the past, ethical interpretations and legal precedents stated that patients were expected to receive complete resuscitation, unless there was a clear statement expressing the opposite [3].

Correspondence to: Nezar Ahmed Salim MSN, BSN, RN, Dubai Health Authority (DHA), Dubai hospital, United Arab Emirates, Dubai, Tel: 00971524818304; E-mail: Nezar_Dubai30@yahoo.com

Key words: Do Not Resuscitate, Terminally ill patient, and Cancer Patient, Allowed Natural Death

Received: October 10, 2017; Accepted: October 30, 2017; Published: October 31,2017 
This paper examines the ongoing and controversial debate regarding the DNR orders. It discusses the rights of terminally ill patients who opt to refuse treatment as well as the various legal and moral ramifications surrounding this particular topic. This argumentative paper delves into the views, policies and cost control measures and other related issues regarding DNR policies. It also analyzes the existing yet contradicting laws, which give patients the right to accept or refuse care yet do not encompass the rights of those actually delivering the care.

\section{Background}

Do Not Resuscitate (DNR) is a medical instruction, ordered by a physician or written in an Advance Directive (AD), by the patients to direct healthcare providers, not to perform CPR if he/she stops breathing or his/her heart no longer beats. The advance directives (ADs) are oral or written declaration in which people state their treatment preferences once they lose decision-making ability, they incorporate withholding or withdrawing medical interventions [4]. This is specifically for CPR and it does not encompass instructions for other healthcare interventions, such as nutrition, pain control, and medications. When CPR is performed, it can reverse premature death, yet it also prolongs terminal illness, worsens discomfort, and exhausts resources [3].

The self-determination act of 1990 established the right of a patient in certain situations where they may be unable to make crucial medical decisions because of incapacitation [5]. Orders given by the patient instruct medical personnel not to perform life saving measures such as cardiopulmonary resuscitation. Truog and Burns provided a comprehensive review of the 40 -year history of (DNR) order published in the New England Journal of Medicine, according to the authors, the journal was among the first to discuss the issue of DNR in 1976, and ever since it has become one of the hot topics in medical ethics and most argued subject among ethical dilemmas [6].

The aim of the DNR order is to permit patients who are dying, to die with integrity without going through futile CPR attempts before being deceased. Many studies proposed that age is an influential determinant of survival post CPR, whereas in many studies the poorer outcome was related to attributing disease rather than age itself. However, in practice in the medical field, there are numerous situations when the line between the recoverable and the non-recoverable state is vague. Circumstances of End Stage Renal Disease (ESRD) or terminal cancer render DNR decision more obvious choice in contrast to other diseases, yet it is still considered a burdensome decision. Generally, in the time of the end-of-life decision-making course, the patient's autonomy is not well obtained [7].

In United States, DNR decision has become legal after the mid of 1970s. . The American Medical Association initially suggested that decisions to refrain from resuscitation to be properly documented. Moreover, it was highlighted that CPR was meant for the prevention of an impetuous death not the treatment of an irreversible or terminal disease [8].

According to the Hospice Patients Aalliance, patients and their families become terrified of the terminology used by health care providers including physicians, nurses and other health care providers as their terminology can be difficult and insensitive. Do Not Resuscitate is an example and so replacing DNR with a kinder terminology; Allow Natural Death “AND” is suggested. Medical field staff mostly are aware that DNR does not mean care is no longer provided. What DNR implies 'simply that goal of treatment has changed', this fact may not appear so to patients and their families since emotions are key element.
The purpose of Allow Natural Death terminology is to assure that only comfort care is provided. By using 'AND', physicians and other health care providers will be acknowledging that the dying patient shall receive the best comfort measures ("Hospice Patients Alliance", 2017).

\section{Methodology}

In this argumentative In this argumentative paper, a search was conducted using conducted using the electronic databases of CINAHL, Medline, PubMed, EPSCO, Science Direct for articles published between 1994 to 2015 by using keywords: "DNR", “Terminally ill patient", "cancer patient", “AND”. Twenty articles were found after extensive searching via electronic databases, which met the inclusion criteria. There is no ethical approval to this article.

\section{Inclusion criteria}

1. Search was limited to English-language

2. Research article

3. Opinion article

4. Integrative literature review

\section{Prevalence of DNR throughout the world}

The DNR decision-making process varies in different countries. The acceptance of the concept of DNR also varies among countries, with most studies conducted in intensive care units and Palliative Care Units (PCUs) [9]. In Western countries, there are more frequent studies regarding the DNR. A study conducted in Pennsylvania, involving 184,057 subjects in 149 acute care hospitals, reported that the percentage of patient proxy who signed DNR consents was ranged between 1-37\% [10]. In Australia, Canada, Sweden, and USA, a study revealed that the DNR rate order among 15 ICUs was $11 \%$ [11]. Approximately $60-70 \%$ of the patients who have DNR consent are those receiving palliative care in USA and Germany [12]. More surprisingly, a study reported that $72 \%$ of Finnish physicians discussing the DNR with the patients whom able to communicate [13].

Usually, during the end-of-life decision-making process, the patient's autonomy is not well procured. However, among many subjects of end-of-life decision, proxy decision making is especially frequent in DNR decisions. A similar trend is also seen in other Asian countries. In Japan, most of the cancer patients are rarely involved in DNR decision making at a teaching hospital [14]. In Japan another study found that $76 \%$ of terminal cancer patients in PCUs signed a DNR consent form [15]. According to Liu and his colleagues the DNR orders are written for $64.4 \%$ of Chinese terminal cancer patients, the order seldom signed by the patient [16].

\section{The argument}

This paper concentrates on the inevitable process of dying, among the patients whom death is inevitable due to an advanced illness, for instance; multi-organ system failure, terminal cancer, end-stage renal, lung, heart or liver disease. However, it can be ambiguous to know the precise time when a patient leaps from distressfully sick to unquestionably departing their life, once the edge is crossed, the inevitability of death becomes clear to a physician who is well trained and experienced. When a human ceases to live, the final event is the cardiac arrest; yet it is not the cause of death, it is the mechanism of death. Efforts made at resuscitation mostly are futile in terms of treating the underlying cause of death, which remains imminent. For this group of patients, the principle of 'Allow Natural Death (AND)' appears highly approachable [17]. 
When CPR is successful, heartbeat and breathing is restored which allows to resume previous lifestyle. The success of CPR depends on the patient's overall medical condition; age alone does not guarantee that CPR will be successful as other illnesses and co-morbidities that go along with age often make CPR less successful. When patients are terminally ill, CPR may not work or may only partially work, leaving the patient brain-damaged or in a worse medical state than before the heart stopped. In these cases, some patients prefer to be cared for without aggressive efforts at resuscitation upon their death [18].

Everyone has the right to receive treatment in life-threatening situations. In such cases, if a person is unconscious or a minor, there is no need to wait to obtain consent from the proxy or guardian, the physicians or nurses should do his or her best to save the life. In these situations if the patient survives, he/she mostly resumes the previous quality of life. On the other hand, with a terminally-ill cancer patient who has undergone all treatment modalities and facing imminent death, futile efforts to save the patient's life will extend his suffering as well as his loved ones. In addition to this, it also affects the patient and his family socially, economically and psychologically. In the event that the terminally ill patient survives and is connected to mechanical ventilator and vasopressor with irreversible brain death, he/she is not expected to return to the previous quality of life.

\section{Legal aspect}

It was declared by the American Heart Association that CPR does not apply to all patients. As As death is an inevitable end, a patient with an irreversible or terminal disease does not necessarily mean to resort to CPR [19]. In 2014, the American Nurses Association and American Society of Anesthesiologists announced a consensus in regard to support patients' rights to self-decision. These rights encompasses that according to the law a capable patient can decline life-saving medical interventions as long as they completely comprehend the ramifications of their choice and allow natural death without resuscitative attempts. Also they concluded that whoever among health care providers attempt to proceed with CPR on a patient against her/his will, it should be considered as violation to the patient's legal right of self-determination. Therefore the DNR order manifests the patient's decision and desire as the legal and medical document aiming to evade life sustaining efforts [20].

It is absolutely pivotal to use the proper words as language can have major impact; whether using AND or DNR, it can crucially affects a major decision for the terminally ill, patient's family as well as for healthcare providers. There is a great advantage for healthcare professionals to contemplate a proper and structured discussion of DNR or AND to rise up orderly a complacent quality end-of-life care, therefore an emphasis should be applied to the use of language and appropriate terminology [21].

Most of healthcare professionals in oncology settings acknowledge that patients with advanced stage cancer have poor survival rates after a cardiac or pulmonary arrest. As a result, formidable efforts have been achieved to raise applying the DNR code in patients with terminal cancer in order to avoid unnecessary and distressing resuscitation [22].

A number of studies supported the DNR order to for terminally ill patients to allow them to pass away in peace [23]. Also CPR in many cases may not end up with direct clinical benefits as the resuscitation could not be fruitful or lead to complications, which in result will extend suffering without treating the underlying illness. Principle factors influencing the use of DNR order include: patients' preferences, survival probability, and expected quality of life prior and after the diagnosis [24-26].

\section{Conclusion}

General questions about meaning and value of suffering should lead us to review the suffering caused by prolonged illness to an individual's personal and family life. Closely related to such consideration on the part of the sick person is whether the unbearable circumstances caused by one's interminable illness makes existence worthwhile at all. Denial of death at some point becomes a delusion, which makes it much harder when it comes to the end-of-life decisions. Beneath these concerns remains a deeper question about the quality of life that individuals and society regard as worth preserving. The DNR is an issue, which is widely debatable with many opinions and conflicts accompanying it. The authors believe that every person who would like to give a point of view on this topic should first be involved in the End of Life Care (E-OL-C). Most of the authors who are opposed to DNR are not involved in clinical practice. It should be taken into consideration especially with patients who have exhausted all other kinds of treatment modalities in which there is multi-organ failure and the hope for a cure has been faded. It should also be considered with the consensus of three qualified consulting doctors that it is in the patient's best interests and benefit to allow natural death to take its course.

The DNR as a concept may appear harsh and unacceptable for the patient and his loved ones, but the use of this term or language plays an important role. The authors strongly believe that it would be more appropriate if the wording is changed from "Do Not Resuscitate" to "Allow Natural Death". If a healthcare provider informs the family that treatment will no longer be given in case of cardiac arrest, they might later regret why they accepted the DNR and think that something else could have been done. In this situation, the concept of "Allow Natural Death" would be more acceptable to them. In a situation that an emotionally charged topic like DNR is the center of discussion, the role of using proper language seems critical and can be considered as a life changing decision from several aspects (patient, family, healthcare providers). In this argument, the authors mainly discussed the DNR from a legal and ethical standpoint, without taking religious views into consideration. The authors believe in God and his potential to create miracles, however for the purpose of this paper, DNR is discussed from a scientific and medical facet only.

\section{Acknowledgment}

The author would like to acknowledge Mr. Nayef Adel Salameh \& Ms. Huda Sarhan for their contribution.

\section{References}

1. Ellis J, Hartley C (2007) Nursing in today's world: trends, issues, and management (9th Ed.). Philadelphia: Lippincott \& Wilkins.

2. Baek MS, Koh Y, Hong S, Lim C, Huh JW (2016) Effect of timing of do-not-resuscitate orders on the clinical outcome of critically ill patients. The Korean Journal of Critical Care Medicine 31: 229-235.

3. Santonocito C, Ristagno G, Gullo A, Weil MH (2013) Do-not-resuscitate order: a view throughout the world. J Crit Care 28: 14-21. [Crossref]

4. Ito H, Nakajima T, Itai K, Ito M, Imai T (2009) On a principal of advance directives. Gan To Kagaku Ryoho 36 Suppl 1: 66-68. [Crossref]

5. Cotler MP (2000) The "do not resuscitate" order; clinical and ethical rationale and implications. Med Law 19: 623-633. [Crossref]

6. Burns JP, Truog RD (2016) The DNR Order after 40 Years. N Engl J Med 375: 504 506. [Crossref]

7. Trivedi S (2013) Physician perspectives on resuscitation status and DNR order in elderly cancer patients. Rep Pract Oncol Radiother 18: 53-56. 
8. National Conference on Cardiopulmonary Resuscitation (CPR) Emergency Cardiac Care (ECC) (1986) Standards and guidelines for cardiopulmonary resuscitation (CPR) and emergency cardiac care (ECC). Part VIII: medicolegal considerations and recommendations. JAMA 255: 2979-2984.

9. Norton SA, Hogan LA, Holloway RG, Temkin-Greener H, Buckley MJ, et al. (2007) Proactive palliative care in the medical intensive care unit: effects on length of stay for selected high risk patients. Critical Care Medicine 35: 1530-1535.

10. Tabak YP, Johannes RS, Silber JH, Kurtz SG (2005) Should do-not resuscitate status be included as a mortality risk adjustor? The impact of DNR variations on performance reporting. Medical Care 43(7):658-666.

11. Cook DJ, Guyatt G, Rocker G, Sjokvist P, Weaver B, et al. (2001) Cardiopulmonary resuscitation directives on admission to intensive-care unit: an international observational study. Lancet 358: 1941-1945.

12. Becker G, Sarhatlic R, Olschewski M, Xander C, Momm F, et al. (2007) End-of-life care in hospital: current practice and potentials for improvement. J Pain Symptom Manage 33: 711-719

13. Hildén HM1, Louhiala P, Palo J (2004) End of life decisions: attitudes of Finnish physicians. J Med Ethics 30: 362-365. [Crossref]

14. Tokuda Y, Nakazato N, Tamaki K (2004) Evaluation of end of life care in cancer patients at a teaching hospital in Japan. J Med Ethics 30: 264-267.

15. Kizawa Y, Tsuneto S, Hamano J, Nagaoka H, Maeno T, et al. (2013) Advance directives and do-not-resuscitate orders among patients with terminal cancer in palliative care units in Japan: a nationwide survey. Am J Hosp Palliat Care 30: 664-669. [Crossref]
16. Liu JM, Lin WC, Chen YM, et al. (1999) The status of the do-not resuscitate order in Chinese clinical trial patients in a cancer center. J Med Ethics 25: 309-314.

17. Taylor RM, Gustin JL, Wells-DiGregorio SM (2010) Improving do-not-resuscitate discussions: A framework for physicians. J Support Oncol 8: 42. [Crossref]

18. Putzel E, Hilleshein K. Bonamigo E (2016) Terminally ill patients' do not resuscitate orders from the doctors' perspective. Scielo Journal Rev Bioét 24.

19. Selekman J, Bochenek J, Lukens M (2013) Children with chronic conditions.

20. Stecher J (2008) ‘Allow natural death' vs. 'do not resuscitate'. Am J Nurs 108: 11. [Crossref]

21. Breault JL (2011) DNR, DNAR, or AND? is language important? Ochsner J 11: 302 [Crossref]

22. Azad AA, Siow SF, Tafreshi A, Moran J, Franco M (2014) Discharge patterns, survival outcomes, and changes in clinical management of hospitalized adult patients with cancer with a Do-Not-Resuscitate Order. J Palliat Med 17: 776-781.

23. Oh DY, Kim JE, Lee CH, Lim JS, Jung KH, et al. (2004) Discrepancies among patients, family members, and physicians in Korea in terms of values regarding the withholding of treatment from patients with terminal malignancies. Cancer 100: 1961-1966.

24. Sham CO, Cheng YW, Ho KW, Lai PH, Lo LW, et al. (2007) Do-not resuscitate decision: The attitudes of medical and non-medical students. J Med Ethics 33: 261-265.

25. Hinkka H, Kosunen E, Metsänoja R, Lammi UK, Kellokumpu-Lehtinen P (2001) To resuscitate or not: a dilemma in terminal cancer care. Resuscitation 49: 289-297. [Crossref]

26. New Designation for Allowing a Natural Death (“A.N.D.”) would Eliminate Confusion and Suffering When Patients are Resuscitated Against their Wishes. http://www. hospicepatients.org/and.html.

Copyright: $(02017$ Salim NM. This is an open-access article distributed under the terms of the Creative Commons Attribution License, which permits unrestricted use, distribution, and reproduction in any medium, provided the original author and source are credited. 